\title{
Nerve Blocks in the Treatment of Headache
}

\author{
Morris Levin \\ Department of Neurology, Dartmouth Hitchcock Medical Center, Lebanon, New Hampshire 03756
}

Summary: Nerve blocks and neurostimulation are reasonable therapeutic options in patients with head and neck neuralgias. In addition, these peripheral nerve procedures can also be effective in primary headache disorders, such as migraine and cluster headaches. Nerve blocks for headaches are generally accomplished by using small subcutaneous injections of amide-type local anesthetics, such as lidocaine and bupivicaine. Targets include the greater occipital nerve, lesser occipital nerve, auriculotemporal nerve, supratrochlear and supraorbital nerves, sphenopalatine ganglion, cervical spinal roots, and facet joints of the upper cervical spine. Although definitive studies examining the usefulness of nerve blocks are lacking, reports suggest that this area deserves further attention in the hope of acquiring evidence of effectiveness. Key Words: Nerve blocks, occipital nerve, greater occipital nerve, occipital neuralgia.

\section{INTRODUCTION AND HISTORY}

The currently available pharmaceutical agents aimed at alleviating acute headaches or preventing headaches can fall short of success in some patients. For some, the pharmaceutical approaches may be hazardous due to any of a number of factors including concomitant cardiovascular, cerebrovascular, or peripheral vascular disease, hepatic or renal disease, pregnancy, or psychiatric conditions. In other patients, medication interactions may prove insurmountable. There is also a subgroup of patients who are simply refractory to traditional modes of therapy. ${ }^{1}$ For many of these patients, peripheral nerve procedures, such as nerve blocks and nerve stimulation, can be dramatically effective and should be considered.

At this time, local anesthetic procedures have unfortunately not been well studied in controlled sham placebo trials. One study ${ }^{2}$ did compare the effectiveness of pericranial nerve blocks to cognitive therapy in cases of chronic head and neck pain. The results were not striking and the numbers of subjects in each group who completed the study were low. In addition, classification of headache type was not clear. Adding to the difficulty of assessing these procedures, it is well known that injections for pain carry a significant placebo effect. ${ }^{3}$

The rationale behind the use of peripheral nerve blockade for pain suppression is based on the ability of local

Address correspondence and reprint requests to: Morris Levin, M.D., Department of Neurology, Dartmouth Hitchcock Medical Center, 1 Medical Center Dr, Lebanon, New Hampshire 03756. E-mail: mo. levin@hitchcock.org. anesthetics to selectively block sensory fibers (sparing motor function) in mixed nerves at relatively low concentrations. The duration of the block depends on the dose and the pharmacokinetic properties of the particular local anesthetic(s), but a longer than expected duration of all or some benefits has been a common observation. Moreover, blockade of a number of nerves in the head and neck can produce beneficial effects in pain syndromes involving regions outside the territory served by the anesthetized nerve. This is often explained by the concept of convergence in the nociceptive system of the head and neck (see as follows), but not all observations can be accounted for by this mechanism.

\section{LOCAL ANESTHETIC BLOCKADE: OVERVIEW OF PHARMACOLOGY AND GENERAL TECHNIQUES}

Local anesthetics (LAs) are divided into the ester and amide categories. They are all weak bases and are available as salts to promote stability and solubility. All LAs have hydrophilic as well as lipophilic components, which makes them easily absorbable and able to cross nerve membranes, respectively. The ester LAs were discovered and used first, but these are also more allergenic, because a metabolite of all ester LAs is the allergen para-amino butyric acid. Ester LAs include procaine and cocaine and tend to be shorter acting than the amide LAs. Amide LAs include lidocaine, mepivacaine, bupivacaine, and prilocaine. Amide LAs tend to be hypoallergenic and well tolerated, hence why they are more commonly used. 
LAs inhibit neural activity by interfering with sodium and potassium currents, thus preventing depolarization. This mechanism is probably based on their entry into neurons and binding to voltage-gated sodium channels, altering their function. The degree of diffusion of LA agents across nerve membranes is related to myelin thickness. Thus, LAs tend to diffuse much better into C fibers than into A fibers, sparing motor function (in typical nerve block doses). All LAs are eventually absorbed systemically, which is how clinical adverse effects generally arise. The most severe effects include the occurrence of seizures or alterations in consciousness when systemic levels of anesthetic agents are high. Adverse cardiac conduction effects can occur with systemic absorption of high doses of LAs. Generally these serious consequences can be avoided by taking measures aimed at avoiding intravascular injection. Technically this is usually not a challenge if one consistently makes sure to pull back the plunger of the syringe and only inject if there is no blood return (implying that the needle tip is not intravascular). However, this is not entirely reliable when using small gauge needles (30 and smaller) or if back pressure is applied too forcefully.

Ester and amide LAs are metabolized differently. Amide LAs are metabolized by the hepatic cytochrome P-450 3A enzyme systems. A number of pharmacologic agents (including anti-arrhythmics, antibiotics, antiepileptics, calcium-channel blockers, beta blockers, and antidepressants) can inhibit this system, leading to increased anesthetic levels due to delayed metabolism; in some situations this could lead to adverse central nervous system effects with only minimal intravascular absorption. Ester LAs are metabolized by hydrolysis, which is a function of the plasma enzyme pseudocholinesterase. Rarely, a patient will have a genetic defect in this enzyme, which leads to elevated anesthetic levels and possible toxicity.

Other adverse effects resulting from LA injection include local infection, nerve damage with later neuroma formation, hematoma (particularly in patients with a bleeding diathesis), and local injury to adjacent structures, depending on the site of injection. Anatomic abnormalities in patients, such as skull defects, local infection, or previous surgical scars, must be identified and avoided. Direct trauma to peripheral nerves is rare, but this can occur. Using small needles, advancing the tip slowly, and aiming for perineural sites are helpful in avoiding adverse effects. Patients will usually report lancinating pain if nerves are directly impinged, at which point the needle should be slightly withdrawn and redirected. In cases in which nerve irritation is believe to have occurred, local measures to reduce inflammation, particularly cold application, are usually sufficient.

Skin injection tends to distort anatomy, so it is important to ascertain geographical markers carefully and even mark the skin appropriately. Because LAs tend to diffuse well throughout the dermis, multiple injection sites are often unnecessary. To gauge the effectiveness of the nerve blockade, sensation testing should reveal an area of anesthesia corresponding to the usual distribution of the nerve(s) injected. If further anesthesia is believed to be indicated, inserting the needle through already anesthetized skin is usually possible and relatively comfortable for the patient.

Commonly used LA agents for local injection include bupivacaine, lidocaine, mepivacaine, and prilocaine, all of which have been used to treat head pain. The latter three have similar potency (approximately one fourth the amount of bupivacaine) and a mid-range duration of action. Lidocaine in the $1 \%$ solution, with an onset of action at approximately 4 to 8 minutes after injection and duration of approximately 1 to 2 hours, is the most common choice. Bupivacaine in the 0.25 or $0.50 \%$ solution offers more prolonged action, with onset in approximately 8 to 12 minutes and duration between 4 and 8 hours. However, bupivicaine is said to be the most cardiotoxic LA. Many choose to combine lidocaine with bupivacaine, generally in a 1:1 ratio. Formulations with epinephrine (used in surgical procedures to reduce local bleeding) are neither necessary nor recommended for nerve blocks. Some practitioners add a corticosteroid medication (although this has no proven benefit). Corticosteroid choices include triamcinolone or methylprednisolone. Ashkenazi et al. ${ }^{4}$ in 2008 reported a randomized double blind control study to address the usefulness of steroid addition to LA injections. The authors found that there was no difference in outcome between patients given greater occipital nerve blocks for headache with either 1) lidocaine and bupivicaine alone or 2) the same agents with the addition of a steroid medication; the severity of their headache and related symptoms were not significantly different between the two groups. Longterm benefits were also similar between the two groups.

\section{SPECIFIC NERVE BLOCKS - TECHNIQUES AND EFFICACY}

\section{Greater occipital nerve block}

The greater occipital nerve (GON) is the primary branch of the second cervical root and innervates the scalp from the level of the external occipital protuberance to the vertex. The GON is located approximately two thirds of the distance on a line drawn from the center of the mastoid to the external occipital protuberance (FIG. 1). The GON is adjacent to the occipital artery and can also be located by palpating for this artery. Injecting approximately $2 \mathrm{~cm}$ lateral to the external occipital protuberance is another useful approach.

Approximately 2 to $3 \mathrm{cc}$ of bupivacaine or lidocaine, or a mixture of the two, injected in the area of the GON 


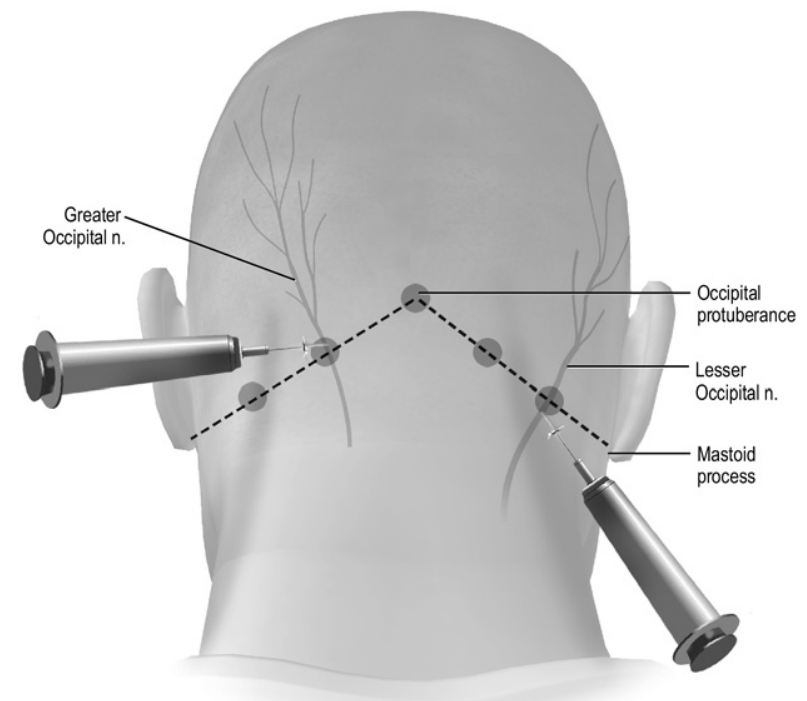

FIG. 1. Greater and lesser occipital nerve blocks. Reproduced with permission from Oxford University Press. (Ashkenazi A, Levin M. Nerve Blocks and Other Procedures for Headache. In: Silberstein SD, Lipton RB, Dalessio DJ, editors. Wolff's Headache and Other Head Pain, 8th ed. Oxford University Press; 2008.)

should be sufficient to anesthetize this nerve. Several unusual adverse effects of GON block have been reported, including local alopecia ${ }^{5}$ and Cushing's syndrome. $^{6}$

The two conditions commonly treated with GON blocks are occipital neuralgia (or "neuritis") and socalled cervicogenic headache. Occipital neuralgia is defined by the International Classification of Headache Disorders, 2nd Edition ${ }^{7}$ as: 1) stabbing, with or without aching, pain roughly in the distribution of the GON, 2) tenderness over the nerve, and 3) reduction of pain by blockade of the GON. A retrospective study of 184 patients with occipital neuralgia showed long-lasting improvement after GON steroid injections with a mean of 31 days of relief. ${ }^{8}$ Other studies have supported the efficacy of this technique. Cervicogenic headache is also defined in the International Classification of Headache Disorders, 2nd Edition. ${ }^{7}$ However, the cervicogenic headache is more controversial with no clear diagnostic tool. Criteria for cervicogenic headache include: 1) head pain referred from a source in the neck, 2) clinical or imaging evidence for a cervical spinal lesion, and 3) abolition of the headache after neural blockade of the cervical pain generator. The GON block was particularly effective in patients with cervicogenic headache in a study of 52 patients with either cervicogenic, migraine, or tension-type headache. ${ }^{9}$ In another study, 100 patients diagnosed with cervicogenic headaches all responded to repeated GON blocks. ${ }^{10}$

GON block seems to be effective in many patients with migraine, although controlled studies have not been done. In a study of 19 patients with acute migraine and allodynia, headache was relieved in 17 , and allodynia was decreased in all patients. ${ }^{11}$ Another study of 25 migraine patients found that $60 \%$ of subjects had significant improvement of migraine pain within 5 minutes of injection. ${ }^{12}$ Long-lasting relief was seen in 26 of 54 migraine patients who received a unilateral GON block with lidocaine and methylprednisolone. ${ }^{13}$

GON blockade seems to be effective in the acute, and possibly prophylactic, treatment of cluster headache $(\mathrm{CH})$. In a study of $14 \mathrm{CH}$ patients treated with GON block, 4 had a good response and 5 had a moderate response. ${ }^{14}$ In a double-blind, placebo-controlled study of GON block ipsilateral to the pain side in $\mathrm{CH}$ patients, Ambrosini et al. ${ }^{15}$ found that $80 \%$ of the treated group responded and none in the placebo (saline injection) group improved. Most patients maintained the effect for at least 4 weeks. Afridi et al. ${ }^{13}$ found that 12 of $22 \mathrm{CH}$ patients responded to GON block, and the response generally lasted for weeks. In the same study, tenderness around the GON seemed to be predictive of a good response to GON block in both the migraine and $\mathrm{CH}$ patients, although the degree of scalp anesthesia obtained after the procedure was not predictive of a good response.

Chronic daily headache $(\mathrm{CDH})$ is a perennially challenging subgroup of patients, particularly in specialty headache and pain clinics. Many fail to respond to numerous pharmacological and nonpharmacological approaches. A placebo-controlled study of the benefits of GON block in chronic daily headache was positive. ${ }^{4}$ Further study is warranted here, as intractable chronic daily headache is a significant health problem with little proven therapy.

GON block has also been successful in other headache types. Matute ${ }^{16}$ in 2008 reported successful GON block in postlumbar puncture headache. Weatherall ${ }^{17}$ reported response to GON block in refractory trigeminal neuralgia. Refractory hemi-crania continua may also respond to GON. ${ }^{18}$ Interestingly, chronic tension type headache seems not to respond to GON block. ${ }^{19} \mathrm{Hecht}^{20}$ found a level of success of GON block in a small series of patients with postconcussive patients.

Tobin and Flitman ${ }^{21}$ looked at the factors that predicted the effectiveness of GON block, including headache type and concurrent medication use. They conducted a chart review of all patients receiving GON block during a 2-year period on those patients whose follow-up data were available (108 patients), and they found impressive response rates in patients with postconcussive syndrome, occipital neuralgia, and migraine. ${ }^{21}$ However, frequent use of symptomatic (analgesic) medication tripled the risk of GON block failure, which the authors $^{21}$ postulated was due to the fact that medication overuse headache does not respond to GON block. The 


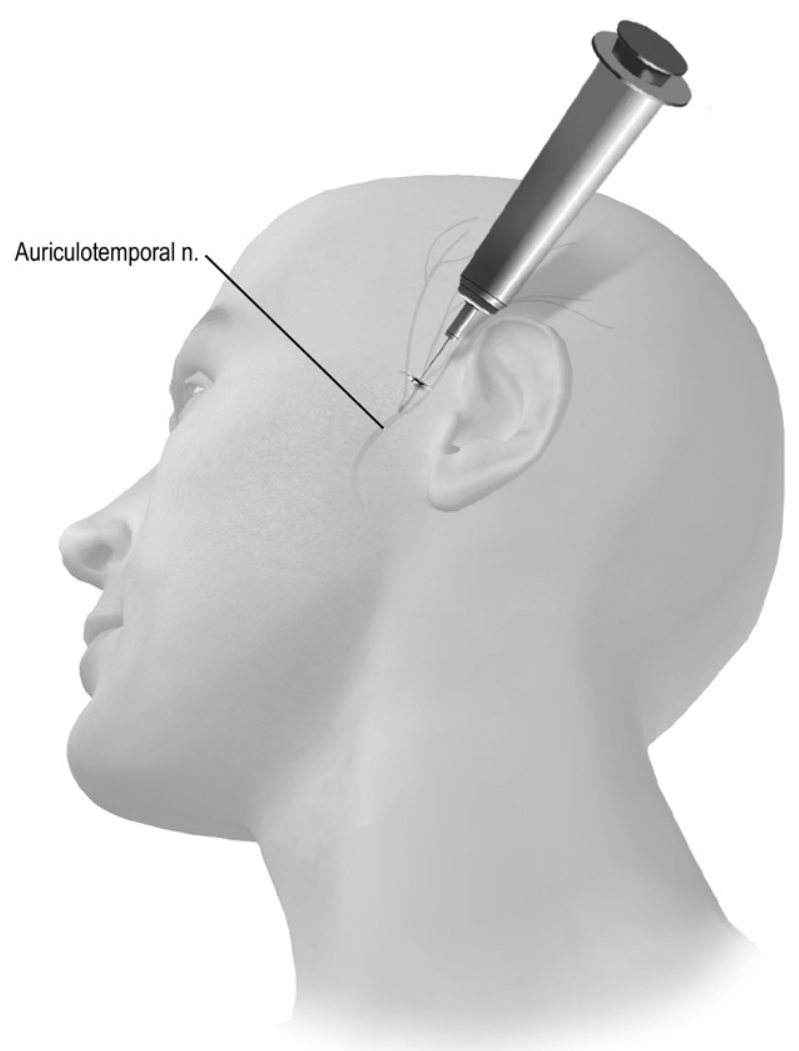

FIG. 2. Auriculotemporal nerve block. Reproduced with permission from Oxford University Press.

reduced effectiveness of GON block was particularly marked in migraineurs, which the authors ${ }^{21}$ similarly speculated was due to migraines being especially susceptible to medication overuse headache.

\section{Lesser occipital nerve block}

The lesser occipital nerve is primarily derived from the cervical plexus (second and third cervical roots) and supplies the inferior scalp and skin of the upper posterior neck. This nerve can be blockaded by injecting approximately 2 to $3 \mathrm{cc}$ of bupivacaine or lidocaine, or a mixture of the two, one third of the way on a line drawn from the center of the mastoid to the greater occipital protuberance (FIG. 1). There are no data supporting the use of lesser occipital nerve blockade in headache disorders, but there are many reports of successful treatments using trigger point injection therapy in the region of the lesser occipital nerve, probably involving some degree of nerve blockade.

\section{Auriculotemporal nerve block}

The auriculotemporal nerve is a branch of the mandibular division of the trigeminal nerve and supplies sensation over the ear and temporalis muscle. Blockade can be done by injecting approximately 2 to $3 \mathrm{cc}$ of bupivacaine and/or lidocaine, superior to the posterior portion of the zygoma just anterior to the ear (FIG. 2). If this block is successful, anesthesia is obtained over the temporal fossa. Although evidence for the effectiveness of auriculotemporal nerve blockade in any headache disorder is presently lacking, there are selected patients who have derived significant benefit from it.

\section{Supraorbital and supratrochlear nerve block}

The supraorbital nerve (SON) and the supratrochlear nerves (STN) are branches of the ophthalmic division of the trigeminal nerve. They both pass through the orbit above the orbital ridge and are easily accessible to neural blockade. The STN is blocked by inserting the needle just above the eyebrow over its medial border and injecting approximately 1 to $2 \mathrm{cc}$ of bupivacaine or lidocaine, or a mixture of both (FIG. 3). To anesthetize the $\mathrm{SON}$, which runs approximately $2 \mathrm{~cm}$ lateral to the supratrochlear nerve, the injection can be done here, or the needle can be advanced laterally through the same puncture that was used for the STN with an injection of 1 to $2 \mathrm{cc}$ of anesthetic (FIG. 3).

The SON and STN are prone to traumatic injury due to their superficial locations, and headaches after frontal trauma with pain and/or tenderness localized to the area should raise a high level of suspicion for the diagnosis of neuralgia involving these nerves. Then this can be confirmed (and treated) with SON/STN blockade. Supraorbital neuralgia is defined by the International Classification of Headache Disorders, 2nd Edition ${ }^{7}$ as: 1) pain in the distribution of the SON, 2) tenderness over the nerve in the supraorbital notch, and 3) abolition of pain by

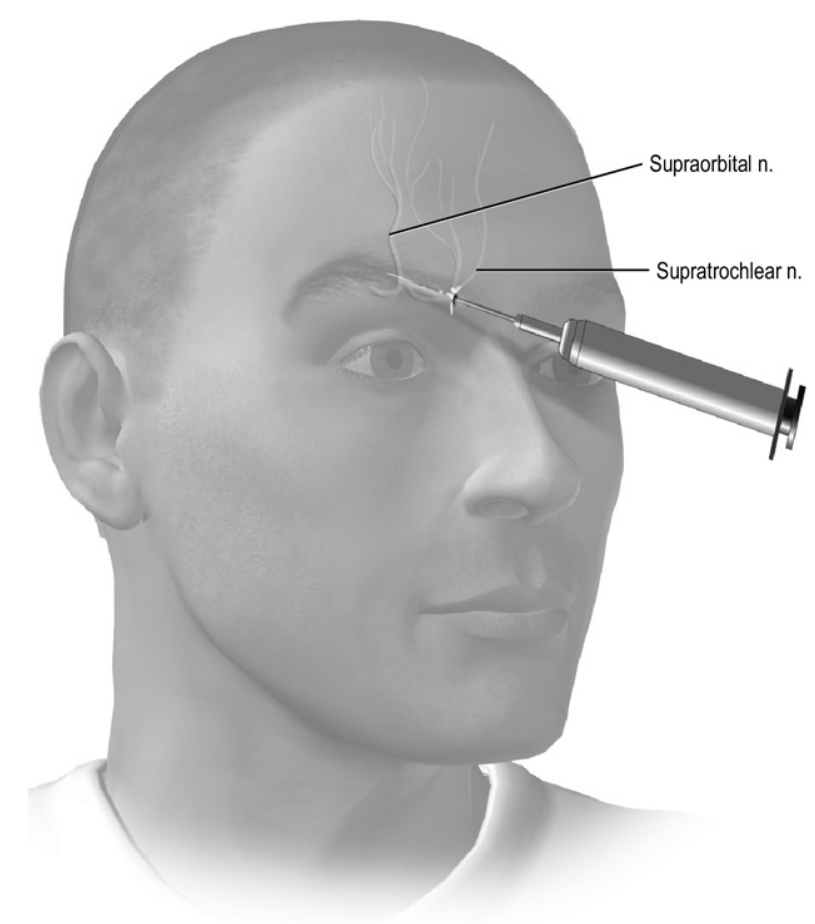

FIG. 3. Suproatrochlear and supraorbital nerve blocks. Reproduced with permission from Oxford University Press. 
blockade or ablation of the SON. ${ }^{22}$ In a study of SON and/or GON blockade in 29 patients with migraine, 25 patients $(85 \%)$ had a favorable response. ${ }^{23}$

\section{Sphenopalatine ganglion blockade}

The sphenopalatine ganglion (or "pterygopalatine ganglion") is a complex region of cell bodies and fibers, including sensory fibers that contribute to the maxillary branch of the trigeminal nerve, as well as both parasympathetic and sympathetic fibers. Anesthesia of the sphenopalatine ganglion can be done through transcutaneous or intraoral injection, but a simpler and less invasive approach is the topical application of local anesthetic to the mucosa overlying it in the lateral wall of the nasal cavity. The procedure is done with the patient supine, with the tip of the nose pointed vertically, and the head turned slightly toward the side of the block. A long, cotton-tipped applicator saturated with $4 \%$ lidocaine is inserted intranasally and applied to the lateral posterior wall of the nasal cavity. This is repeated until pain relief is obtained (FIG. 4). An alternate method involves a flexible small bore catheter placed in the same place (near the posterolateral wall of the nasal cavity) through which lidocaine is slowly dripped until pain relief is obtained.

Sphenopalatine ganglion blockade was initially proposed as an acute treatment option for cluster headache, based on reported acute cluster headache response to cocaine applied to the posterior nasal mucosal region. Results have been generally positive, ${ }^{24-26}$ although in one study of 30 male patients with cluster headaches, the results were mostly negative. ${ }^{27}$ Patients can learn to do

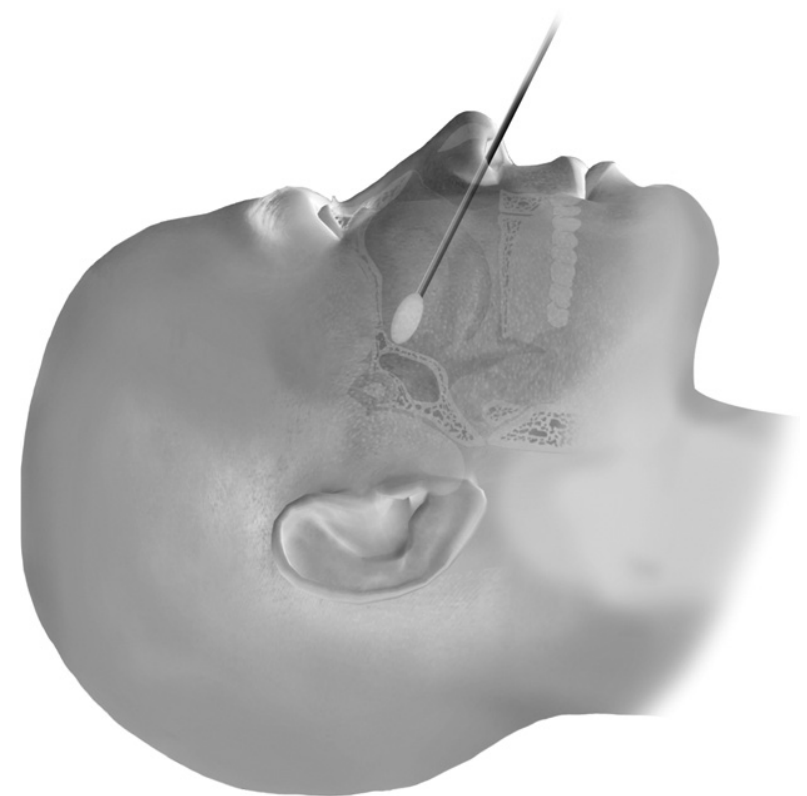

FIG. 4. Sphenopalatine block. Reproduced with permission from Oxford University Press. this technique themselves with very gratifying results in many cases.

\section{Cervical root blockade}

The second cervical root has been proposed as a target in some headaches, particularly those in which GON block was of some benefit. The third cervical root has also been proposed as a target in some headaches, generally those believed to be caused by "whiplash" or other cervical injury or pathology. ${ }^{28}$ A study of 40 patients who had head pain after a whiplash-type injury found a $57 \%$ consistent response rate to anesthetic blockade of the $\mathrm{C} 3$ roots. ${ }^{29}$ These techniques also require fluoroscopy and experienced injectors.

Adverse effects that may occur after root blocks include local hemorrhage, infection, nerve trauma with persistent neuropathic pain, sensory loss, weakness or paresthesias, and injury to vascular structures, such as carotid or vertebral arteries.

\section{Mechanisms of nerve block effects in primary headache}

The mechanisms by which these procedures work are not clear. Migraine and cluster headaches are believed to be centrally mediated primary neuropathic phenomena, and it is unclear how blocking cervical roots or trigeminal nerve branches might affect these processes. Clearly, nociceptive physiology must be modulated at higher levels if these peripheral procedures truly improve the pain of these disorders.

For acute pain relief, the mechanism may stem from a reduction in afferent "tone," leading to a lessening of activity at the level of the trigeminal nucleus caudalis and cervical dorsal horn. Because of the organization of primary and secondary neurons serving head regions, this reduction in overall afferent transmission at the first synapse of the nociceptive pathways governing pain originating in the head and neck could conceivably reduce pain perception in a much broader area than that served by the blocked nerve blocked. There is good evidence that convergence between the cervical and trigeminal systems happens at this level and that changes in one component can modulate the other. $^{30-33}$

But how might long-term improvement result from anesthesia that lasts at most only 4 to 6 hours? Many clinicians have observed that it is crucially important to "break the pain cycle," and this may well correlate with physiological factors key to the production of chronic pain in the first place. It has become clear in recent years that central sensitization at the level of the first nociceptive synapse (dorsal horn or spinal trigeminal nucleus) can often be the primary explanation for persistent neuropathic pain when the source of pain has ended. Perhaps reducing nociceptive traffic through a temporary neural blockade in a sensitized 
system may allow "winding-down" of central sensitization. Ongoing pain on the basis of peripheral sensitization (at the level of peripheral nociceptors) could also be ameliorated through the same process (i.e., blockade of a converging peripheral nerve or nerves may lead to a relatively increased threshold for nociceptive transmission from another converging set of sensitized nociceptors).

When therapeutic anesthetic blocks are used for headaches believed to have stemmed from actual cervical, occipital, or trigeminal nerve injury or dysfunction, such as post-traumatic damage or vascular compression, then mechanisms for pain relief are more transparent; the specific nerves or systems responsible for a peripheral neuropathic pain phenomenon are theoretically being anesthetized. On the other hand, most peripheral neuropathic pain conditions of the head and neck seem to involve some degree of central sensitization, and peripheral blockade should not help dramatically unless, again, its effects exert a modulatory influence on synaptic transmission as well.

\section{CONCLUSIONS}

Greater occipital nerve block, the most widely used local anesthetic procedure in headache conditions, is safe and relatively easy to perform in the office. Adverse effects are few and infrequent. The procedure can result in rapid relief of pain and allodynia, and effects may last for several weeks. Other scalp nerves can be easily blocked as well, including supraorbital, supratrochlear, and auriculotemporal nerves. Although there is limited evidence from controlled studies, peripheral nerve blocks seem to be viable treatment options for selected groups of headache patients, particularly those with intractable headache or facial pain. Local tenderness overlying superficial nerves of the scalp and face might be predictive of good results with local nerve blockade, but this remains to be proven.

Blinded, sham-controlled studies are needed to establish the efficacy of nerve blocks in intractable headache disorders. Challenges include the difficulty in creating valid sham designs and controlling for headache diagnosis and other confounding factors that might influence clinical improvement or lack of benefit.

\section{REFERENCES}

1. Schullman EA, Brahin EJ. Refractory Headache. Headache 2008; 48:770-778.

2. Gale G, Nussbaum D, Rothbart P, Hann B, Leung V, Kanetz G. A randomized treatment study to compare the efficacy of repeated nerve blocks with cognitive therapy for control of chronic head and neck pain. Pain Res Manag 2002;7:185-189.

3. de Craen AJ, Tijssen JG, de GJ, Kleijnen J. Placebo effect in the acute treatment of migraine: subcutaneous placebos are better than oral placebos. J Neurol 2000;247:183-188.
4. Ashkenazi A, Matro R, Shaw JW, Abbas MA, Silberstein SD. Greater occipital nerve block using local anaesthetics alone or with triamcinolone for transformed migraine: a randomised comparative study. J Neurol Neurosurg Psychiatry 2008;79:415-417.

5. Shields KG, Levy MJ, Goadsby PJ. Alopecia and cutaneous atrophy after greater occipital nerve infiltration with corticosteroid. Neurology 2004;63:2193-2194.

6. Lavin PJ, Workman R. Cushing syndrome induced by serial occipital nerve blocks containing corticosteroids. Headache 2001;41: 902-904.

7. Headache Classification Committee. The International Classification of Headache Disorders, 2nd edition. Cephalalgia 2004;24:1160.

8. Anthony M. Headache and the greater occipital nerve. Clin Neurol Neurosurg 1992;94:297-301.

9. Bovim G, Sand T. Cervicogenic headache, migraine without aura and tension-type headache. Diagnostic blockade of greater occipital and supra-orbital nerves. Pain 1992;51:43-48.

10. Rothbart P, Fiedler K, Gale GD, Nussbaum D, Hendlerb N. A descriptive study of 100 patients undergoing palliative nerve blocks for chronic intractable headache and neck ache. Pain Res Management 2000;5:243-248.

11. Ashkenazi A, Young WB. The effects of greater occipital nerve block and trigger point injection on brush allodynia and pain in migraine. Headache 2005;45:350-354.

12. Cook BL, Malik SN, Shaw JW, Oshinsky ML, Young WB. Greater occipital nerve (GON) block successfully treats migraine within five minutes. Neurology 2006;66:A42 (abstract).

13. Afridi SK, Shields KG, Bhola R, Goadsby PJ. Greater occipital nerve injection in primary headache syndromes - prolonged effects from a single injection. Pain 2006;122:126-129.

14. Peres MF, Stiles MA, Siow HC, Rozen TD, Young WB, Silberstein SD. Greater occipital nerve blockade for cluster headache. Cephalalgia 2002;22:520-522.

15. Ambrosini A, Vandenheede M, Rossi P, et al. Suboccipital injection with a mixture of rapid- and long-acting steroids in cluster headache: a double-blind placebo-controlled study. Pain 2005;118: 92-96.

16. Matute E. Bilateral greater occipital nerve block for post-dural puncture headache. Anaesthesia 2008;63:557-558.

17. Weatherall MW. Idiopathic trigeminal neuropathy may respond to greater occipital nerve injection. Cephalalgia 2008;28:664666.

18. Rozen T. Cessation of hemiplegic migraine auras with greater occipital nerve blockade. Headache 2007;47:917-919.

19. Leinisch-Dahlke E, Jurgens T, Bogdahn U, Jakob W, May A Greater occipital nerve block is ineffective in chronic tension type headache. Cephalalgia 2005;25:704-708.

20. Hecht JS. Occipital nerve blocks in postconcussive headaches: a retrospective review and report of ten patients. J Head Trauma Rehabil 2004;19:58-71.

21. Tobin, JA, Flitman, SS. Occipital nerve blocks: effect of symptomatic medication overuse and headache type on failure rate headache. Headache 2009;49:1479-1485.

22. Sjaastad O, Stolt-Nielsen A, Pareja JA, Fredriksen TA, Vincent M. Supraorbital neuralgia. On the clinical manifestations and a possible therapeutic approach. Headache 1999;39:204-212.

23. Caputi CA, Firetto V. Therapeutic blockade of greater occipital and supraorbital nerves in migraine patients. Headache 1997;37: 174-179.

24. Costa A, Pucci E, Antonaci F, et al. The effect of intranasal cocaine and lidocaine on nitroglycerin induced attacks in cluster headache. Cephalalgia 2000;20:85-91.

25. Hardebo JE, Elner A. Nerves and vessels in the pterygopalatine fossa and symptoms of cluster headache. Headache 1987;27:528532.

26. Kittrelle JP, Grouse DS, Seybold ME. Cluster headache. Local anesthetic abortive agents. Arch Neurol 1985;42:496-498.

27. Robbins L. Intranasal lidocaine for cluster headache. Headache 1995;35:83-84.

28. Bogduk N, Marsland A. On the concept of third occipital headache. J Neurol Neurosurg Psychiatry 1986;49:775-780. 
29. Lord S, Barnsley L, Wallis B, Bogduk N. Third occipital headache: a prevalence study. J Neurol Neurosurg Psychiatry 1994;57:1187.

30. Bartsch T, Goadsby PJ. Stimulation of the greater occipital nerve induces increased central excitability of dural afferent input. Brain 2002;125:1496-1509.

31. Bartsch T, Goadsby PJ. Increased responses in trigeminocervical nociceptive neurons to cervical input after stimulation of the dura mater. Brain 2003;126:1801-1813.
32. Busch V, Jakob W, Juergens T, Schulte-Mattler W, Kaube H, May A. Functional connectivity between trigeminal and occipital nerves revealed by occipital nerve blockade and nociceptive blink reflexes. Cephalalgia 2006;26:50-55.

33. Piovesan EJ, Kowacs PA, Tatsui CE, Lange MC, Ribas LC, Werneck LC. Referred pain after painful stimulation of the greater occipital nerve in humans: evidence of convergence of cervical afferents on trigeminal nuclei. Cephalalgia 2001;21:107-109. 